\title{
Strain Effects in Graphene and Graphene Nanoribbons: The Underlying Mechanism
}

\author{
Yang Li ${ }^{1,2}$, Xiaowei Jiang ${ }^{1,2}$, Zhongfan $\mathrm{Liu}^{1}$, and Zhirong $\mathrm{Liu}^{1}(\bowtie)$ \\ ${ }^{1}$ College of Chemistry and Molecular Engineering, State Key Laboratory for Structural Chemistry of Unstable and Stable Species, and \\ Beijing National Laboratory for Molecular Sciences, Peking University, Beijing 100871, China \\ ${ }^{2}$ School of Physics, Peking University, Beijing 100871, China \\ Received: 18 May 2010 / Revised: 9 June 2010 / Accepted: 11 June 2010 \\ (C) The Author(s) 2010. This article is published with open access at Springerlink.com
}

\begin{abstract}
A tight-binding analytic framework is combined with first-principles calculations to reveal the mechanism underlying the strain effects on electronic structures of graphene and graphene nanoribbons (GNRs). It provides a unified and precise formulation of the strain effects under various circumstances-including the shift of the Fermi (Dirac) points, the change in band gap of armchair GNRs with uniaxial strain in a zigzag pattern and its insensitivity to shear strain, and the variation of the $k$-range of edge states in zigzag GNRs under uniaxial and shear strains which determine the gap behavior via the spin polarization interaction.
\end{abstract}

\section{KEYWORDS}

Graphene, graphene nanoribbons (GNRs), band gap, strain, first-principles calculations, tight-binding model

\section{Introduction}

Apart from its remarkable fundamental properties, graphene has attracted intensive interest in recent years as a promising candidate material for the new generation of electronics and spintronics $[1,2]$. To construct a graphene-based device or circuitry, the ability to modulate the electronic structure of graphene is definitely required. Possible means to achieve this goal include patterning [3], electric field effects [4], and chemical doping [5]. Recently, it was even proposed that strain can be utilized to generate various basic elements for all-graphene electronics [6].

Growth of graphene on substrates with a different lattice constant usually introduces strain which can be detected by Raman spectroscopy $[7,8]$. In some cases, relief of the compressive strain will result in the formation of ridges and buckling [9]. Even in the absence of a lattice mismatch, strain still arises along the edges and manifests some interesting quantum features $[10,11]$. Graphene is the strongest material ever measured [12]. Small [13, 14] or large (up to $30 \%$ ) [15] strains can be readily exerted on graphene in labs. Recently, by carefully controlling the uniaxial and shear strains, Bao et al. succeeded in creating periodic ripples in graphene sheets [16].

Strain may have important effects on the electronic properties of a material. Strain in $\mathrm{Si}, \mathrm{SiGe}$, and $\mathrm{Ge}$ has been successfully employed in the semiconductor industry to improve the mobility of field-effect transistors (FETs) [17]. For carbon nanotubes, it has been established that the band structure can be dramatically altered by strain $[18,19]$. Considering the close relation between graphene and carbon nanotubes, 
one would expect strain will have important influence on the electronic structure of graphene. Scanning tunneling microscopy (STM) studies on graphene have indeed revealed a correlation between local strain and tunneling conductance [20]. Theoretically, both the tight-binding (TB) model and ab initio approaches have been widely adopted to investigate the effect of strain on the band structure of graphene and graphene nanoribbons (GNRs) [14, 21-35]. It was shown that below a threshold value (which may exceed $20 \%$, depending on direction), uniaxial strain will not open an energy gap for infinite graphene sheets, but just cause the Fermi crossing to move away from the $K$ point $[26,27,30,31]$. In the case of GNRs, uniaxial strain has little influence on the band structure of zigzag GNRs (ZGNRs), while the energy gap of armchair GNRs (AGNRs) is modified in a periodic way with a zigzag pattern [21, 25, 29, 32]. Accordingly, AGNRs and ZGNRs possess distinct charge transport properties under strain [30, 32-34], e.g., ZGNRs remain robust against high uniaxial strain [33]. The effect of strain on the chemical reactivity of graphene has also been investigated [36]. Very recently, a TB calculation was conducted to investigate the effects of both uniaxial and shear strains on GNRs, and an analytical solution at $k=0$ was obtained to provide physical insights into understanding the response of AGNRs [35].

Although there have been many studies of the strain effects in graphene and GNRs, most of them have focused on uniaxial strain, while the effect of shear strain has seldom been studied (with the exception of the recent work by $\mathrm{Lu}$ and Guo [35]). Furthermore, although various effects of strain on graphene and GNRs have been revealed, a universal theory that can provide a concise physical understanding of the different simulation results from $\mathrm{TB}$ and $a b$ initio approaches is still lacking. In this paper, we calculate the band structures of graphene and GNRs under both uniaxial and shear strain using density functional theory (DFT), and formulate a universal explanation for the various effects of strain.

\section{Method and model}

\subsection{First-principles calculations}

The uniform strain in graphene can be generally written as a strain tensor:

$$
\varepsilon=\left(\begin{array}{cc}
\varepsilon_{\mathrm{A}} & \gamma \\
0 & \varepsilon_{\mathrm{Z}}
\end{array}\right)
$$

where $\varepsilon_{\mathrm{A}}$ and $\varepsilon_{\mathrm{Z}}$ are the uniaxial strain along the armchair and zigzag directions, respectively, and $\gamma$ is the shear strain. A positive value of $\varepsilon_{\mathrm{A}}\left(\varepsilon_{\mathrm{Z}}\right)$ means the strain is elongating (tensile) while a negative value corresponds to compressive strain. In our first-principles calculations, we consider three special cases where we keep one of $\varepsilon_{\mathrm{A}^{\prime}} \varepsilon_{\mathrm{Z}}$, and $\gamma$ at a fixed value while the other two are relaxed to achieve the lowest total energy.

For GNRs, we define $x$-direction to be along the unstrained ribbon axis and $y$ as the transverse direction (Fig. 1). In the presence of a uniaxial or shear strain, the $x$-component of the coordinate of the $i$-th atom changes according to following relations:

$$
\begin{cases}x_{i} \rightarrow\left(1+\varepsilon_{\mathrm{A} / Z}\right) x_{i}, & \text { (Uniaxial) } \\ x_{i} \rightarrow x_{i}+\gamma y_{i}, & \text { (Shear) }\end{cases}
$$

where $\varepsilon_{\mathrm{AZ}}$ denotes $\varepsilon_{\mathrm{A}}$ in AGNRs and $\varepsilon_{\mathrm{Z}}$ in ZGNRs. The $y$-component of the atom coordinates is free to relax. Two examples are illustrated in Fig. 1 to show the deformation.

We performed our DFT calculations implemented with the Vienna ab initio simulation package (VASP) [37]. The projector augmented wave (PAW) pseudopotential [38], and the general gradient approximation (GGA) PW91 [39] were adopted with a $520 \mathrm{eV}$ cutoff energy. We used a supercell geometry in which adjacent sheets are separated by $10 \AA$ to avoid any interaction between them. The Monkhorst-Pack grid mesh was $45 \times 45$ for graphene and $25 \times 1$ for GNRs. Energy was taken to be converged when the difference between consecutive total energy was less than $0.1 \mathrm{meV}$. Atoms were relaxed using the conjugate gradient method until the forces on atoms were less than $0.01 \mathrm{eV} / \AA$.

\subsection{Analytic model}

In order to analyze the DFT calculations, we adopted the theory presented by Yang and Han, which is based on the Hückel TB model and an analytic framework [18]. When a strain is exerted on graphene, real space vectors are transformed according to $r=(I+\varepsilon) r_{0}$, where $I$ is the unit matrix and $\varepsilon$ the strain tensor. Subscript 


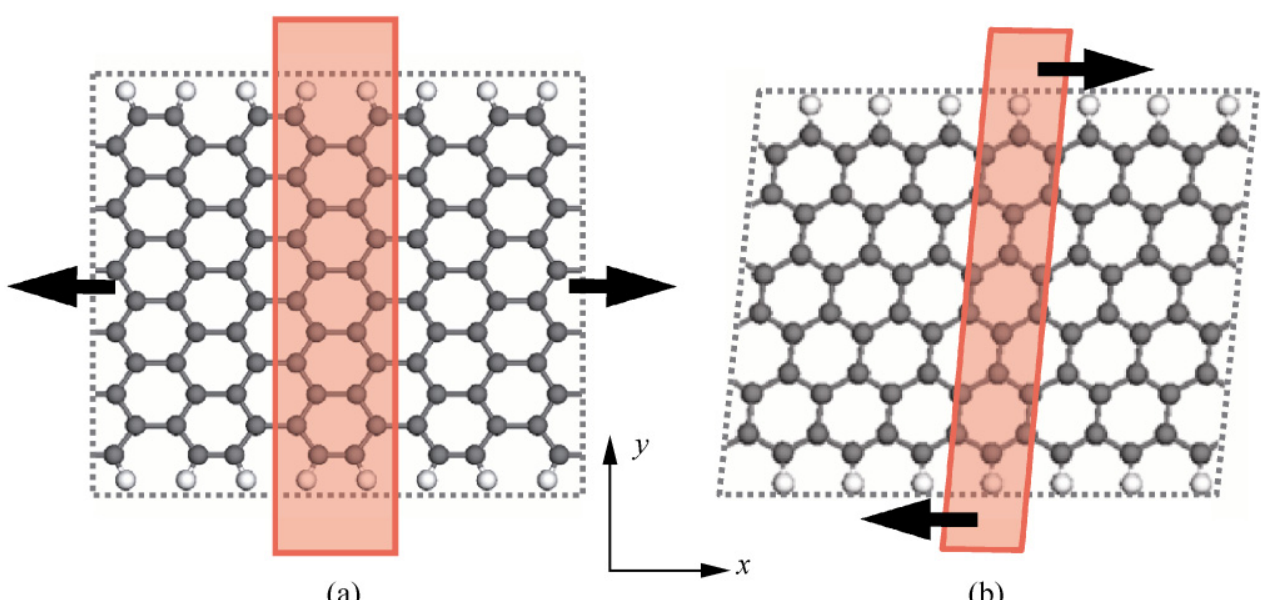

(a)

(b)

Figure 1 Schematic views of GNRs under strain: (a) AGNRs with ribbon width $N_{\mathrm{A}}=12$ under a uniaxial strain of $\varepsilon_{\mathrm{A}}=10 \%$; (b) ZGNRs with ribbon width $N_{\mathrm{Z}}=6$ under a shear strain of $\gamma=10 \%$. The edge carbon atoms (gray circles) are passivated by hydrogen atoms (white circles). The shaded rectangles denote the unit cells of the systems

" 0 " denotes undeformed states. The resulting Brillouin zone (BZ) for the reciprocal vector $k$ in the deformed space is no longer a regular hexagon (Fig. 2(a)) [22-24, $26,27]$. By introducing a new quantity

$$
\boldsymbol{k}^{*}=(\boldsymbol{I}+\boldsymbol{\varepsilon})^{\mathrm{T}} \boldsymbol{k}
$$

the BZ in the $\boldsymbol{k}^{*}$ space will be restored to hexagonal as in the undeformed case since

$$
\boldsymbol{k} \cdot \boldsymbol{r}=\boldsymbol{k} \cdot(\boldsymbol{I}+\boldsymbol{\varepsilon}) \boldsymbol{r}_{0}=\left[(\boldsymbol{I}+\boldsymbol{\varepsilon})^{\mathrm{T}} \boldsymbol{k}\right] \cdot \boldsymbol{r}_{0}=\boldsymbol{k}^{*} \cdot \boldsymbol{r}_{0}
$$

$k^{*}$ can be regarded as the undeformed reciprocal vector. The introduction of $k^{*}$ and the property in Eq. (4) greatly facilitate the analysis. For example, the TB Hamiltonian becomes

$$
H(\boldsymbol{k})=\sum_{i=1,2,3} t_{i} \exp \left(\mathrm{i} \boldsymbol{k} \cdot \boldsymbol{a}_{i}\right)=\sum_{i=1,2,3} t_{i} \exp \left(\boldsymbol{k}^{*} \cdot \boldsymbol{a}_{i 0}\right)
$$

where the $\boldsymbol{a}_{i}$ are three bond vectors from a carbon atom, and $t_{i}$ are hopping parameters which depend on the bond length. This incorporates the effect of strain into the variation of $t_{i}$. When a symmetric strain is applied, $t_{1}=t_{2}=t_{3}$, and the Fermi points (synonymous with the Dirac points in this paper) $k_{\mathrm{F}}$, defined by $E\left(\boldsymbol{k}_{\mathrm{F}}\right)=\left|H\left(\boldsymbol{k}_{\mathrm{F}}\right)\right|=0$, sit at the vertex of the hexagonal BZ, i.e., the $K$ points in Fig. 2. For uniaxial and shear strains which are asymmetric, $t_{1}, t_{2}$, and $t_{3}$ are no longer equal, and the Fermi points will deviate from
$K$ in the $\boldsymbol{k}^{*}$ space. Under the linear approximation, the deviation $\Delta \boldsymbol{k}_{\mathrm{F}}^{*}=\boldsymbol{k}_{\mathrm{F}}^{*}-\boldsymbol{k}_{\mathrm{K}}^{*}$ is determined to be [18]

$$
\left\{\begin{array}{l}
\Delta k_{\mathrm{F} x}^{*} a_{0}=S_{t}[(1+v) \sigma \cos 3 \theta+\gamma \sin 3 \theta] \\
\Delta k_{\mathrm{F} y}^{*} a_{0}=S_{t}[-(1+v) \sigma \sin 3 \theta+\gamma \cos 3 \theta]
\end{array}\right.
$$

where $\Delta k_{\mathrm{F} x}^{*}$ and $\Delta k_{\mathrm{F} y}^{*}$ are the components of $\Delta \boldsymbol{k}_{\mathrm{F}}^{*}$ along $x$ and $y$ directions, $\theta$ is the angle between the $x$ axis and the zigzag direction, $\sigma$ is the uniaxial strain along the $y$ direction, and $v$ is the Poisson's ratio: i.e., (positive) $\sigma$ is the elongating strain along the $y$ direction while $v \sigma$ is the compressive strain along the $x$ direction. $S_{t}$ is a constant introduced here to reflect the dependence of $t$ on $a$ :

$$
S_{t}=-\left.\frac{a}{2 t} \frac{\mathrm{d} t}{\mathrm{~d} a}\right|_{a=a_{0}}
$$

where $a_{0}$ is the equilibrium bond length. For the Harrison hopping parameter relation $t_{i}=t_{0}\left(a_{0} / a_{i}\right)^{2}$ [40], $S_{t}=1.0$. The deviation of $K^{\prime}$ is opposite to that of $K$. The dispersion relation of deformed graphene is given by expanding $E(k)$ near the Fermi points:

$$
E\left(\boldsymbol{k}^{*}\right)= \pm \frac{3}{2} t_{0} a_{0}\left|\boldsymbol{k}^{*}-\boldsymbol{k}_{\mathrm{F}}^{*}\right|
$$

Thus the effect of (small) strain is to move the energy cone in the $k^{*}$ space (Fig. 2(b)). 


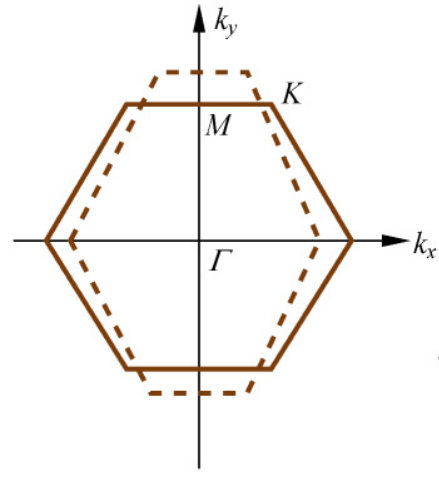

(a) (b)

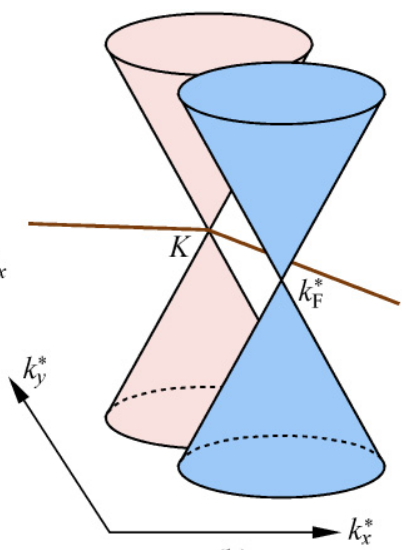

Figure 2 Schematic illustrations of the effects of strain: (a) deformation of the Brillouin zone; (b) shift of the energy cone away from $K$ points in the $\boldsymbol{k}^{*}$ space

The electronic properties of GNRs are analyzed based on the above results for graphene with the consideration that the $k$ values of the allowed electronic states lie on some discrete lines due to the quantum confinement effect.

\section{Results and discussion}

\subsection{Graphene: The shift of the Fermi points}

We investigated the band structure of infinite graphene sheets under various strains. For a shear of $5 \%$, our DFT calculation shows that the conduction band energy at the $K$ point is not equal to the valence band energy, and there is a small gap of $\sim 100 \mathrm{meV}$. However, similar to studies of the uniaxial case [26, 27, 30, 31, 41], this does not mean that a band gap is opened by strain. By scanning the vicinity of $K$ along various $k$ lines, we find that the valence and conduction bands touch at a point deviating from $K$ (Fig. 3(a)), and thus the system remains gapless. The dispersion is still well described by a cone. A global fit (solid lines) gives a hopping parameter $t_{0}=2.67 \mathrm{eV}$, which is consistent with the relaxed equilibrium value $(\sim 2.7 \mathrm{eV})$ in the literature $[18,42]$. We also tested the influence of the uniaxial strain along the armchair and zigzag directions, and observed similar behaviors, i.e., the shift of the Fermi points. The main difference between the various strains is the shifting direction, which is summarized in Fig. 3(b). Note that shear strain will shift the Fermi points away from any symmetric $k$ paths. Furthermore, the shifting of Fermi points near the $K$ points is exactly opposite to that near $K^{\prime}$. They may collide if the uniaxial strain is large enough. It was previously suggested using an analytical model that a realistic band gap will be opened under such a collision [43], and a similar effect has also been observed in TB and DFT calculations [26, 30, 31].

The results of our DFT calculations can be satisfactorily explained in the theoretical framework developed by Yang and Han [18] (see Section 2). The shifting direction of Fermi points predicted by Eq. (6)

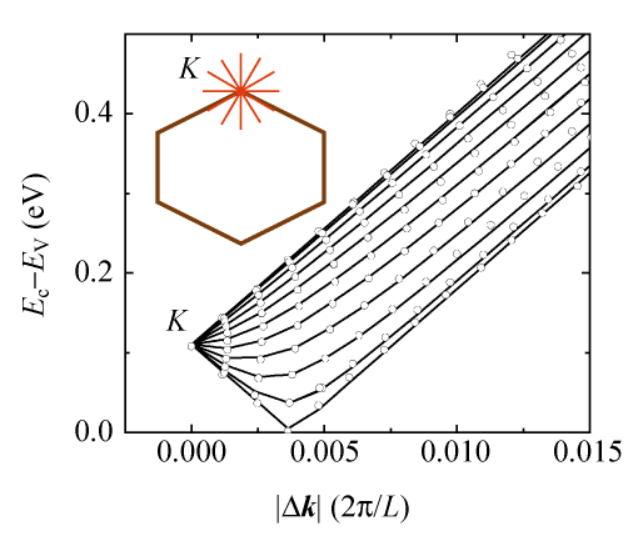

(a)

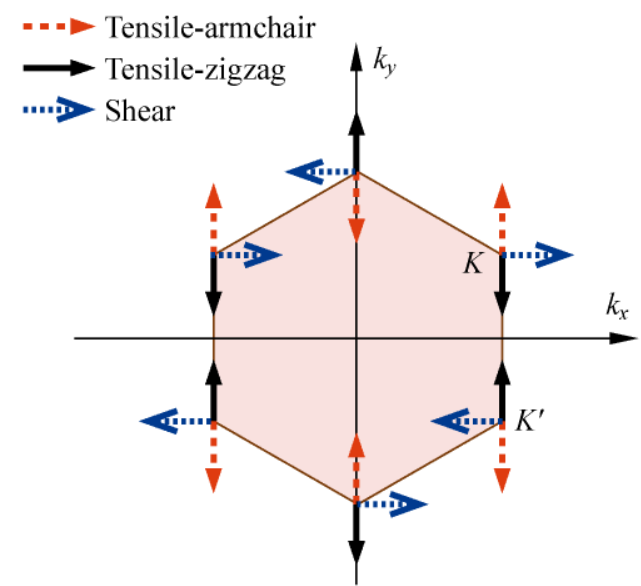

(b)

Figure 3 Shift of Fermi points under strain in graphene. (a) Energy difference between the conduction band and the valence band for various $\boldsymbol{k}$ lines starting from the $K$ point. DFT data under a shear strain of $\gamma=5 \%$ are shown as circles and a global fit is shown as solid lines. The inset is a schematic view of selected $\boldsymbol{k}$ lines. (b) Summary of the shift direction of Fermi points under different types of strain 
is fully consistent with Fig. 3(b). It is also inferred from Eq. (6) that when the strain (uniaxial or shear) rotates by angle $\theta$, the shifting direction of Fermi points will rotate by $2 \theta^{(1)}$, neglecting the slight difference between $\boldsymbol{k}$ and $\boldsymbol{k}^{*}$; this is consistent with the observation of Mohr et al. [27]. By combining Eqs. (6) and (8), we obtain the following analytical expression for the pseudo gap at $K$ and $K^{\prime}$ points in the $k^{*}$ space:

$$
E_{\text {gap }}\left(k_{\mathrm{V}}^{*}\right)= \begin{cases}3 t_{0} S_{t}\left(1+v_{\mathrm{A}}\right) \varepsilon_{\mathrm{A}} & \text { (uniaxial strain along armchair) } \\ 3 t_{0} S_{t}\left(1+v_{\mathrm{Z}}\right) \varepsilon_{\mathrm{Z}} & \text { (uniaxial strain along zigzag) } \\ 3 t_{0} S_{t} \gamma & \text { (shear strain) }\end{cases}
$$

In Fig. 4, we depict the $E_{\text {gap }}\left(\boldsymbol{k}_{\mathrm{V}}^{*}\right)$ data obtained in DFT calculations for various strains up to $5 \%$. The value of $E_{\text {gap }}\left(\boldsymbol{k}_{\mathrm{V}}^{*}\right)$ under shear strain is smaller than that under uniaxial strain. Equation (9) indicates that the difference comes from the Poisson's ratio $v$, i.e., when graphene is stretched in one direction, it will shrink in the perpendicular direction, and thus further shift the Fermi points. Using the averaged $v$ values of $v_{\mathrm{A}}=0.145$ and $v_{Z}=0.16$ in DFT calculations (Fig. 4 , inset), we made a global fit (the solid lines in Fig. 4 ) to the $E_{\text {gap }}\left(\boldsymbol{k}_{\mathrm{V}}^{*}\right)$ data,

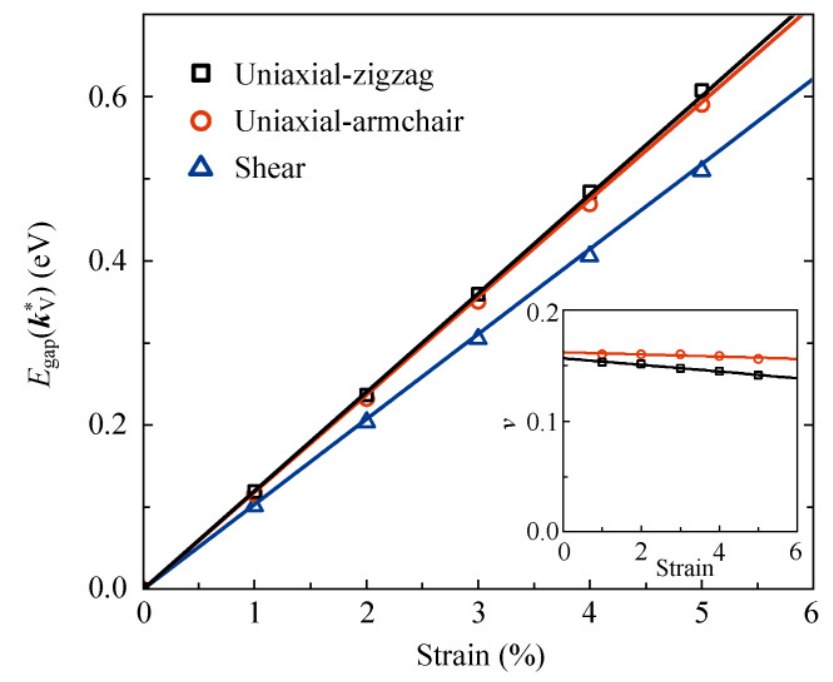

Figure 4 The calculated energy gap at $\boldsymbol{k}_{\mathrm{V}}^{*}$ for various strains. Straight lines are a global fit according to Eq. (9). Inset is the Poisson's ratio $v$ for uniaxial strain along zigzag and armchair directions

(1) Note that the angle $\theta$ between zigzag direction and the $x$-direction should be subtracted from the angle between shifting direction and the $x$-direction ( $3 \theta$ in Eq. (6)) to obtain the desired value. Refer to Fig. 1 of Ref. [18] for a schematic graphic and the result confirms that $v$ indeed accounts for the different effects of shear and uniaxial strains. By adopting $t_{0}=2.67$ as determined above, we obtain $S_{t}=1.29$, which is slightly larger than the value given by the Harrison relation [40] $\left(S_{t}=1.0\right)$, while smaller than the approximation made by Pereira et al. [26] $\left(S_{t} \approx 1.7\right)$.

\subsection{AGNRs: The effects of discrete $k$-lines}

We now examine whether strain can change the energy gap in GNRs where the size confinement may play an important role. It is well known that the confinement effect will open a gap in AGNRs and the gaps can be divided into three groups according to the ribbon width i.e., $N_{\mathrm{A}}=3 p, 3 p+1$, or $3 p+2$, where $p$ is a positive integer $[3,44]$. Our DFT results of the shear effect on AGNRs are summarized in Fig. 5. It can be seen that shear strain makes the band structure a little smoother, but the modification of the band architecture, especially the energy gap, is slight (Fig. 5(a)). Even under a shear strain of up to $\gamma=10 \%$, the variation of the gap is less than $60 \mathrm{meV}$ for a system with $N_{\mathrm{A}}=12$. The influences on the three groups of AGNRs are similar (Fig. 5(b)). The curves of the gap are symmetric with respect to $\gamma=0$, and thus the slope at $\gamma=0$ is exactly equal to zero. Such an insensitive response of AGNRs to the shear strain is consistent with a recent TB calculation by $\mathrm{Lu}$ and Guo [35].

In contrast to the shear case, it has been revealed that uniaxial strain has a significant effect on the electronic structure and conductivity of AGNRs [21, 25, 29, 32, $34,35]$. Uniaxial strain will move some bands towards the Fermi level, while moving the others away [25]. For the purpose of further analysis, we repeated the calculations under uniaxial strain and briefly list the main DFT results in Fig. 6. It was confirmed that the energy gap $E_{\text {gap }}$ was effectively modified in a periodic zigzag pattern, while there are also distinct phases for the three groups of $N_{\mathrm{A}}=3 p, 3 p+1$, and $3 p+2$ (Fig. 6(a)). The slope of $E_{\text {gap }}\left(\varepsilon_{\mathrm{A}}\right)$ is almost identical for various $N_{\mathrm{A}}$ values, while both the peak value and the period of the pattern decrease with increasing $N_{\mathrm{A}}$ (Fig. 6(b)).

The effects of shear and uniaxial strains on AGNRs can be understood by the shift of Fermi points we described in the previous section and the fact $[45,46]$ that the $k$ value of the allowed electronic states of carbon 


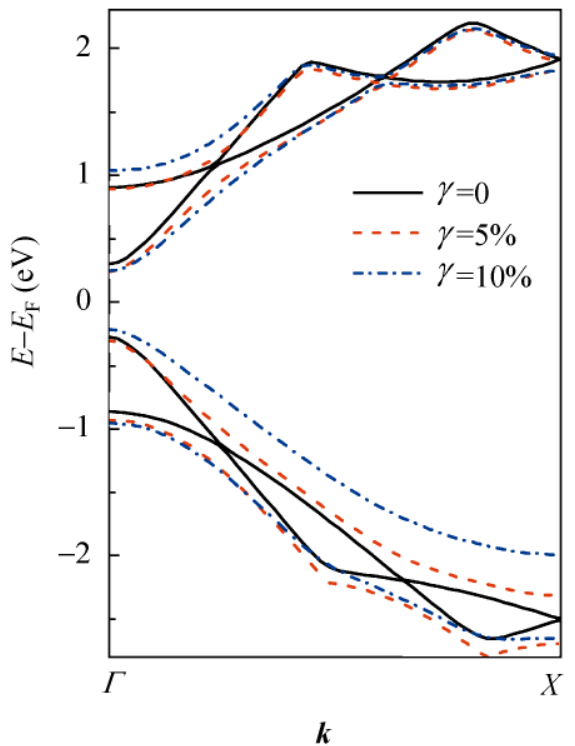

(a)

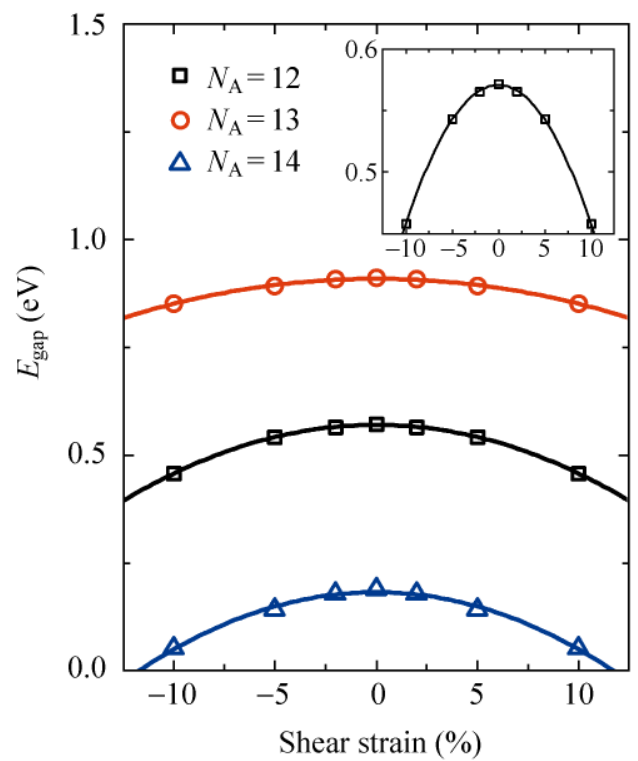

(b)

Figure 5 The effect of shear strain on AGNRs. (a) The electronic structure of AGNRs with a width $N_{\mathrm{A}}=12$ under the shear strain $\gamma=0$, $5 \%, 10 \%$. Only four bands close to the Fermi level are shown. (b) The energy gap as a function of $\gamma$ for AGNRs with $N_{\mathrm{A}}=12,13,14$. Solid lines are a quadratic fitting. Inset is the magnification of the $N_{\mathrm{A}}=12$ case

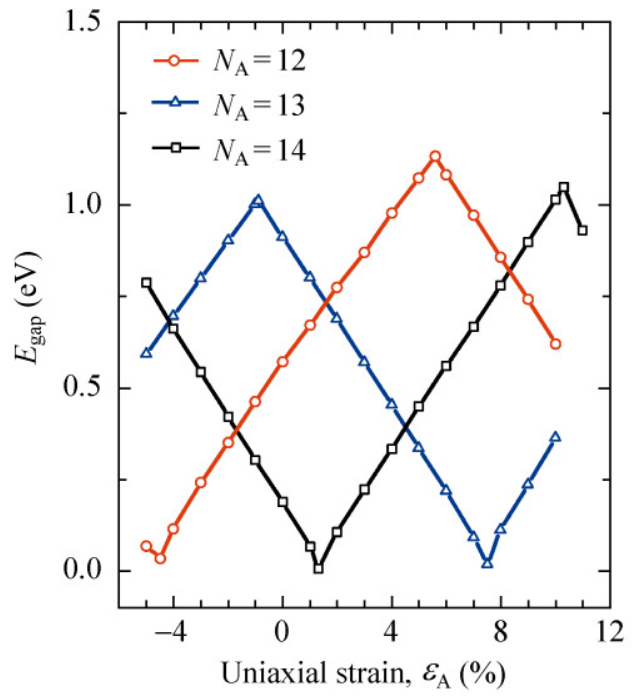

(a)

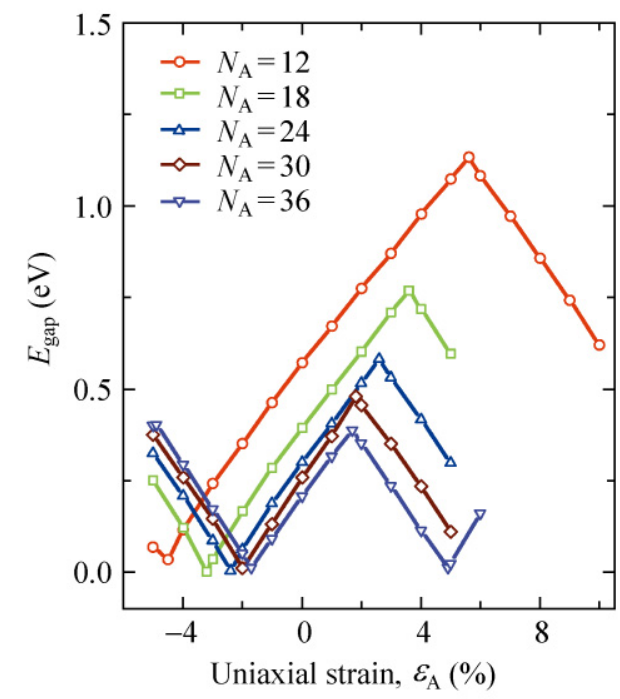

(b)

Figure 6 The calculated energy gap of AGNRs as a function of the uniaxial strain $\varepsilon_{\mathrm{A}}$ : (a) examples for three groups with ribbon widths $N_{\mathrm{A}}=3 p, 3 p+1$, and $3 p+2$; (b) examples for the group $N_{\mathrm{A}}=3 p$

nanotubes and GNRs lie on parallel lines (Fig. 7). The allowed $k$-lines of the three groups of AGNRs have different crossing situations with the $K$ point (Fig. 7(a)), resulting in different energy gaps. When a uniaxial strain is applied, the Fermi points deviate from $K$, moving in a direction perpendicular to the allowed $k$-lines (Fig. 7(b)), and thus make some bands closer (to the Fermi level) while the others move further away. When the Fermi points arrive at the middle of two neighboring $k$-lines (the position " 1 " in Fig. $7(b)$ ), the energy gap reaches its maximum. When the Fermi points land on the $k$-lines (the position " 2 " in Fig. 7(b)), the energy gap is decreased to zero. The interval between neighboring $k$-lines decreases with increasing 


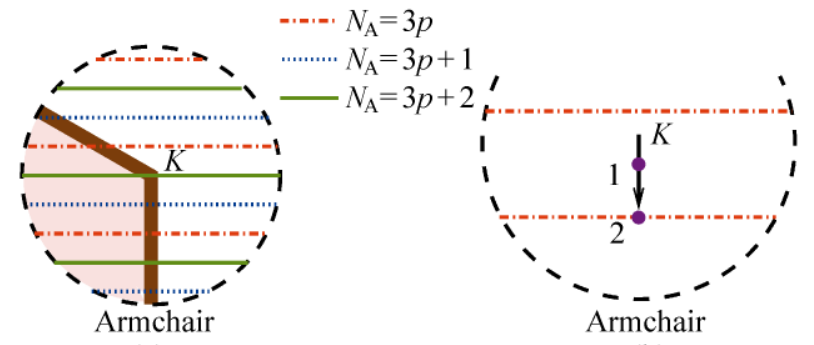

(a)

(b)

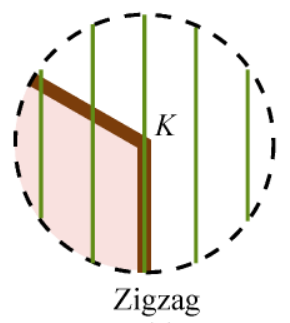

(c)

Figure 7 Schematic parallel $\boldsymbol{k}$-lines for allowed electronic states in (a) and (b) AGNRs and (c) ZGNRs. Thick lines are part of the Brillouin zone boundary with the vertex $K$, and thin lines represent the allowed $\boldsymbol{k}$-lines for GNRs. The line with the arrow in (b) indicates the shift of Fermi point under uniaxial strain

ribbon width of the GNRs, so the maximum gap and the strain required to obtain such a maximum will also decrease. This is the microscopic origin underlying the observations in Fig. 6. For the shear strain, the movement of the Fermi points is parallel to the allowed $\boldsymbol{k}$-lines, and so has no effect on the energy gap under the linear approximation. From Eq. (6) and Fig. 3(b), it can also be noted that the effect of uniaxial elongation along the armchair direction is equivalent to that of compression along the transverse (zigzag) direction, which provides a natural explanation for the observed symmetric change of the gap of AGNRs for uniaxial strains along the nearest-neighbor (armchair) and second nearest neighbor (zigzag) directions [32].

In a recent paper by $\mathrm{Lu}$ and Guo [35], an analytical solution at $k=0$ (the $\Gamma$ point) was obtained for AGNRs with consideration of edge distortion and third nearest neighbor coupling to interpret the response of the band gap to uniaxial and shear strains. In contrast, our approach (considering only nearest neighbor interactions), gives a full analytical dispersion relation near the Fermi points, and interprets the properties of AGNRs by relating them to the higher dimensional state space of graphene. Thus, the underlying origin of the observed properties can be described in the higher space: strains shift the Dirac points, and the energy gap decreases to zero when the Dirac points reach the allowed $\boldsymbol{k}$-lines of AGNRs. This approach is also helpful in revealing the difference between uniaxial and shear strains, e.g., uniaxial and shear strains generally play similar roles (Eq. (6)), while the distinct behavior of shear in not affecting the energy gap is just incidental because it shifts the Fermi points in a direction parallel to the allowed $\boldsymbol{k}$-lines.
The ribbon width plays an important role in the universal scaling properties of nanostructures [44, 47]. According to Eqs. (6), (8) and the allowed $k$-lines determined by the hard-wall boundary condition [48], we obtain an analytic expression for the half-period $\left(\Delta \varepsilon_{\mathrm{A}}\right.$, defined as the strain interval between the neighboring turning points in Fig. 6) and the maximal value of the energy gap of AGNRs under uniaxial strain:

$$
\left\{\begin{array}{l}
\Delta \varepsilon_{\mathrm{A}}=\frac{\pi}{\sqrt{3} S_{t}\left(1+v_{\mathrm{A}}\right)} \cdot \frac{1}{N_{\mathrm{A}}+1} \\
E_{\text {gap }}^{\max }=\frac{\sqrt{3} \pi t_{0}}{N_{\mathrm{A}}+1}
\end{array}\right.
$$

A comparison between the prediction and the DFT calculation is provided in Fig. 8. Parameters obtained in infinite graphene sheets, i.e., $t_{0}=2.67 \mathrm{eV}, v_{\mathrm{A}}=0.145$ and $S_{t}=1.29$, are used in the prediction. Although no direct fitting is applied to the AGNRs, the agreement between the theory and the DFT calculation is excellent. This suggests the edges in AGNRs play a role mainly via the confinement effect. In the work of Sun et al. [25], an empirical formula, $E_{\text {gap }}^{\max }=14.06 / N_{\mathrm{A}}$ was obtained by fitting the calculated data for AGNRs, and the resulting constant 14.06 is very close to the factor $\sqrt{3} \pi t_{0}(=14.5)$ in our prediction. It is also noted that $S_{t}$ affects only $\Delta \varepsilon_{\mathrm{A}}$, but not $E_{\text {gap }}^{\max }$. If the Harrison relation $\left(S_{t}=1.0\right)$ is adopted, then the theoretical prediction (the dotted line in Fig. 8(a)) will overestimate the $\Delta \varepsilon_{\mathrm{A}}$ values.

\subsection{ZGNRs: The effects of edge states}

Given the success in explaining the strain effects on AGNRs in terms of Fermi-point shifting and discrete 


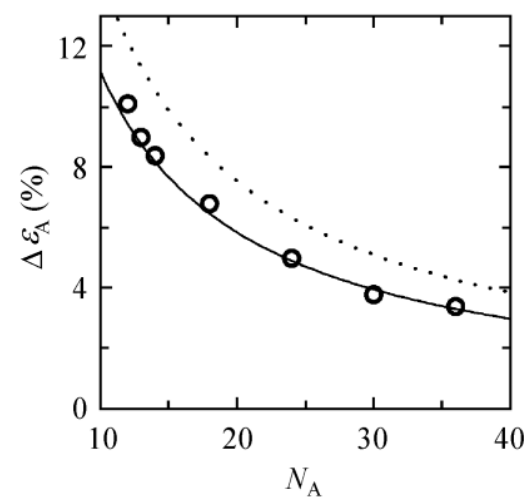

(a)

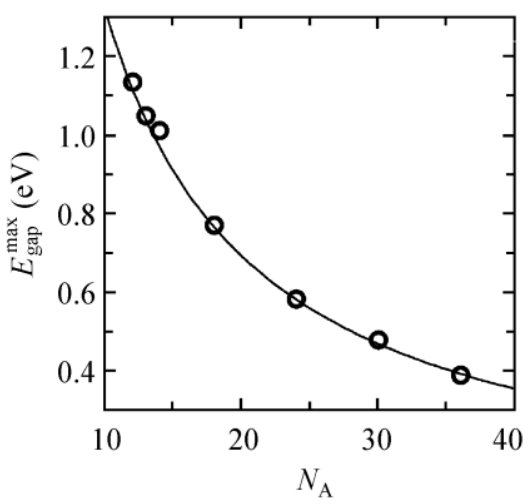

(b)

Figure 8 (a) The half-period $\Delta \varepsilon_{\mathrm{A}}$ and (b) the maximal value $E_{\text {gap }}^{\max }$ of the energy gap under uniaxial strain as a function of the ribbon width of AGNRs. Circles are DFT calculation results while solid lines are the theoretical prediction using Eq. (10) and the dotted line in (a) is the prediction using the Harrison relation $\left(S_{t}=1.0\right)$

$\boldsymbol{k}$-lines, one may expect that ZGNRs obey similar rules. If this is true, shear strain will open an energy gap in ZGNRs while the uniaxial strain will have no effect because the allowed $\boldsymbol{k}$-lines in ZGNRs are perpendicular to those in AGNRs (see Fig. 7). Such a prediction was indeed verified for carbon nanotubes [18]. However, our DFT calculations indicate that this is not the case for ZGNRs (Fig. 9). They show that neither uniaxial nor shear strains can open an energy gap in ZGNRs when spin polarization is not considered (Figs. 9(a) and 9(b)). It has been suggested that a gap will be opened in ZGNRs when spin polarization is considered [49], and the resulting half-metallicity has potential applications in spintronics [49, 50]. Our DFT calculations with spin polarization reveal that the effects of both uniaxial and shear strains on the gap are still weak in this situation (Figs. 9(c) and 9(d)). Another interesting feature is that the $\boldsymbol{k}$-range of the edge states (which appear as the flat curve segments near $E=0$ ) expands under uniaxial strain (Fig. 9(c)) while remains unchanged under shear strain (Fig. 9(d)) (the spin-unpolarized calculations show similar trends, and are not discussed here).

Why do ZGNRs behave abnormally when compared with AGNRs? As indicated by Lu and Guo [35], the key lies in the existence of edge states in ZGNRs (Fig. 10). Following the derivation in Refs. [51, 52], the edge states for infinite width are given by

$$
\phi(m) \mathrm{e}^{\mathrm{i} k(n+1)} t_{1}+\phi(m-1) \mathrm{e}^{\mathrm{i} k(n+1)} t_{2}+\phi(m-1) \mathrm{e}^{\mathrm{i} k n} t_{3}=0
$$

which yields

$$
\phi(m)=\phi_{0}\left(\frac{t_{2}}{t_{1}}+\frac{t_{3}}{t_{1}} \mathrm{e}^{-\mathrm{i} k}\right)^{m}
$$

Thus the condition for the edge states to exist is

$$
\left|\frac{t_{2}}{t_{1}}+\frac{t_{3}}{t_{1}} \mathrm{e}^{-\mathrm{i} k}\right| \leqslant 1
$$

When there is no strain, $t_{1}=t_{2}=t_{3}=t_{0}$, and the critical $k$ value for edge states is

$$
k_{\mathrm{c}}^{(0)}=\frac{2 \pi}{3}
$$

When there is strain, $t_{1}=t_{2}=t_{3}=t_{0}$ is no longer applicable. By linearly expanding $t_{i}$ as in Ref. [18], the deviation of the critical $k$ value for edge states, $\Delta k_{\mathrm{c}}=k_{\mathrm{c}}-k_{\mathrm{c}}^{(0)}$, is determined from Eq. (13) as

$$
\Delta k_{\mathrm{c}}=\sqrt{3}[\sigma(1+v) \cos 2 \theta+\gamma \sin 2 \theta]
$$

For the strain application scheme in DFT calculations on AGNRs, $\theta=90^{\circ}$, so Eq. (15) predicts that $k_{\mathrm{c}}$ will become smaller under uniaxial strain while remaining invariant under shear strain, which is consistent with the results in Fig. 9. A quantitative comparison also 


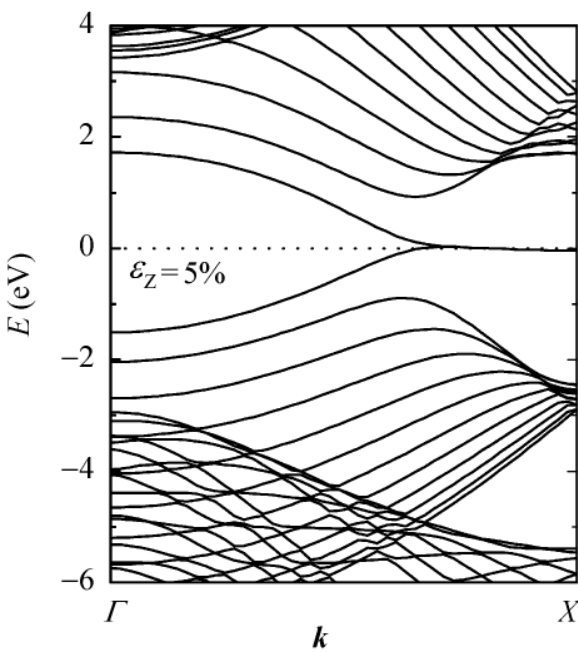

(a)

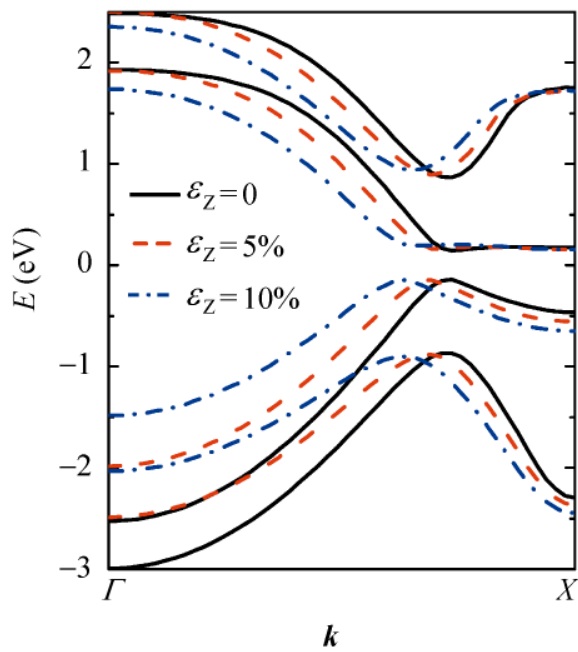

(c)

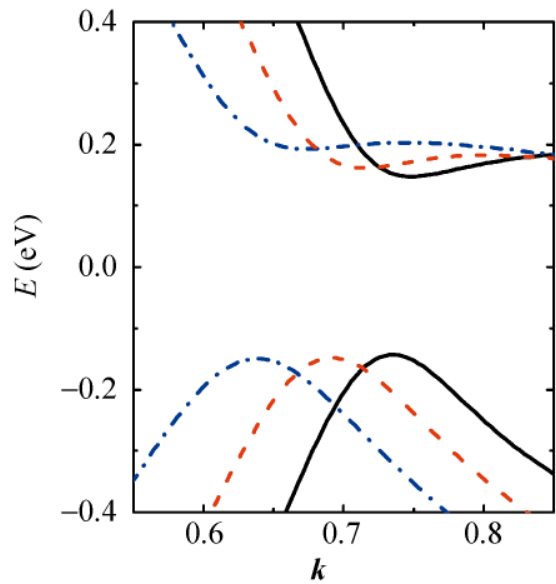

(e)

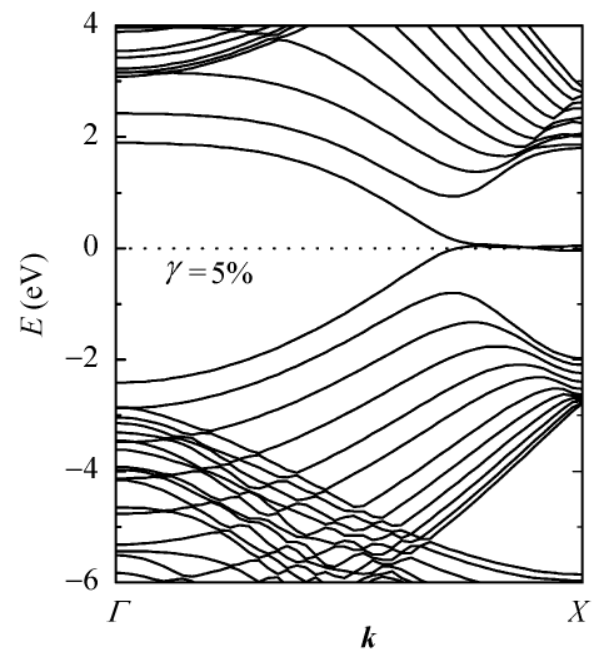

(b)

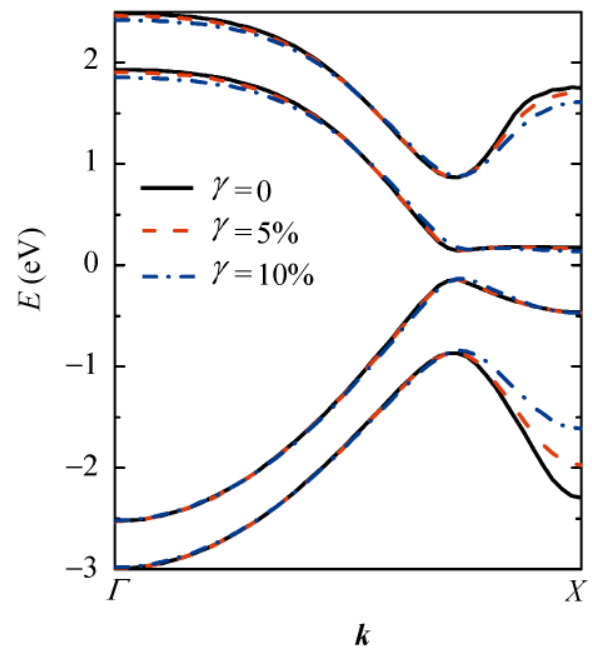

(d)

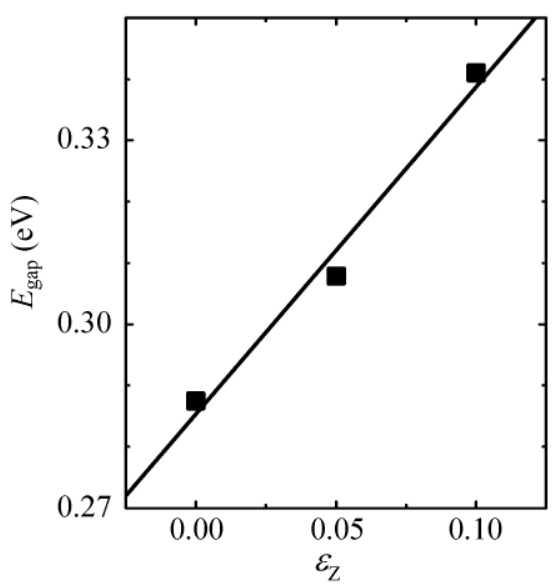

(f)

Figure 9 ((a), (b)) The spin-unpolarized and ((c), (d)) spin-polarized electronic structure of ZGNRs with a width $N_{Z}=12$ under various strains. Only four bands close to the Fermi level are shown in ((c), (d)). (e) A local magnification of (c) to demonstrate the variation of the energy gap. (f) Energy gap as a function of $\varepsilon_{Z}$ under spin polarization, where solid squares are DFT calculation data while the solid line is a linear fit 


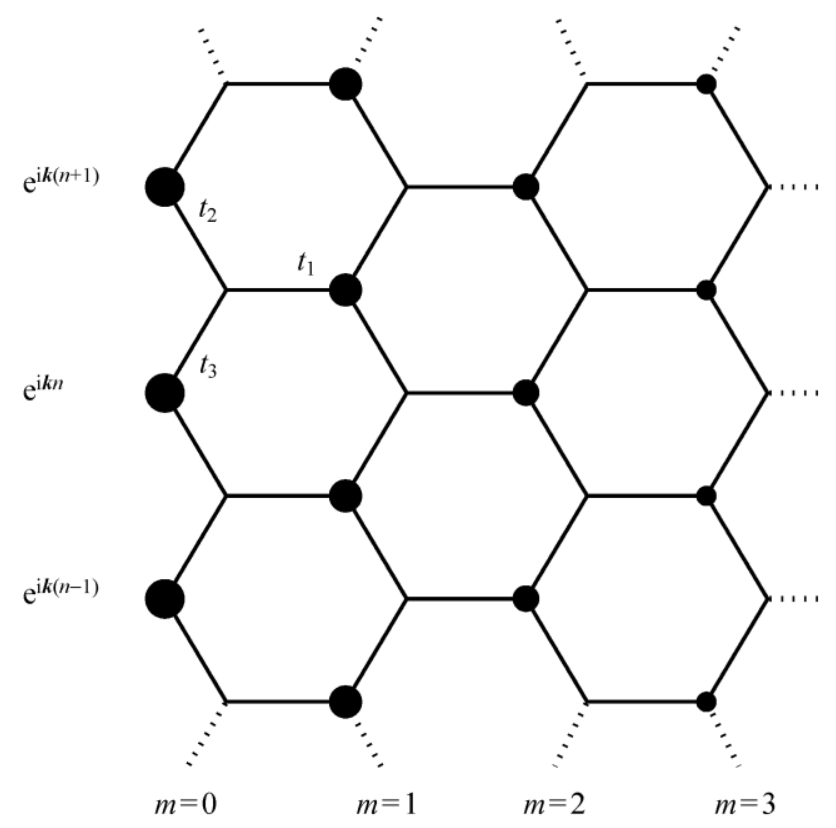

Figure 10 Schematic graphics of edge states in ZGNRs. The magnitude of the wavefunction at each carbon site is indicated by the radius of the circle

confirms the validity of Eq. (15) (data not shown). (Small) strain can modify the $\boldsymbol{k}$-range of edge states, but cannot eliminate their existence, which is the reason why the energy gap of ZGNRs is unaffected by both uniaxial and shear strains. The insensitivity of the gap with respect to strain suggests that ZGNRs may act as ideal conducting wires in graphene electronics.

It was shown in a TB calculation that an energy gap in ZGNRs will be opened up by a tensile strain under spin polarization [35]. A closer inspection of our DFT results confirms such a behavior (Fig. 9(e)), e.g., the gap increases slightly from 0.29 to $0.34 \mathrm{eV}$ when a $10 \%$ uniaxial strain is applied. The separation of the valence and conduction bands in AGNRs is induced by the spin polarization interaction and the band separation at the zone boundary $(X)$ can be approximated as $[35,53]$

$$
E_{\text {gap }}^{X}=U\left(n_{1, \uparrow}-n_{1, \downarrow}\right)
$$

where $U$ describes the strength of the polarization interaction, and $n_{1, \uparrow}\left(n_{1, \downarrow}\right)$ is the spin-up (-down) population on a sublattice. $n_{1, \uparrow}-n_{1, \downarrow}$ is proportional to the population of edge states, and thus proportional to the $k$-range of the edge states. Under the approximation that the relative variation of $E_{\text {gap }}$ under a strain is equal to that of $E_{\text {gap }}^{X}$, we combine Eqs. (15) and (16) and obtain the effect of uniaxial strain on gap in AGNRs as

$$
\frac{\mathrm{d}}{\mathrm{d} \varepsilon_{\mathrm{A}}}\left(\frac{E_{\text {gap }}\left(\varepsilon_{\mathrm{A}}\right)}{E_{\text {gap }}(0)}\right)=\frac{\mathrm{d}}{\mathrm{d} \varepsilon_{\mathrm{A}}}\left(\frac{\pi-k_{\mathrm{c}}}{\pi / 3}\right)=\frac{3 \sqrt{3}}{\pi}\left(1+v_{\mathrm{A}}\right)
$$

Analysis of the DFT gap data (Fig. 9(f)) reveals the existence of a slight curvature, while the slope at $\varepsilon_{\mathrm{A}}=0$ gives a value of $\frac{\mathrm{d}}{\mathrm{d} \varepsilon_{\mathrm{A}}}\left(\frac{E_{\text {gap }}\left(\varepsilon_{\mathrm{A}}\right)}{E_{\text {gap }}(0)}\right)$ of 1.51 , reasonably consistent with the predicted value of $\frac{3 \sqrt{3}}{\pi}\left(1+v_{\mathrm{A}}\right)=1.93$. The theoretical value is also close to the empirical value of 1.6 obtained in the TB calculation of $\mathrm{Lu}$ and Guo [35]. The $k$-range of edge states remains invariant under shear strain, so the energy gap does not change with shear strain under a linear approximation.

\section{Summary}

We have calculated the electronic structures of graphene and GNRs under various uniaxial and shear strains with a first-principles approach, and formulated an underlying mechanism via an analytic TB theory. The effect of strain on graphene is to drive the Fermi points away from the $K$ points while keeping the cone-like energy dispersion, and thus does not open an energy gap. The energy gap of AGNRs is insensitive to shear strain, but is modified by uniaxial stain in a zigzag pattern, which is caused by the moving of the graphene Fermi points between discrete $\boldsymbol{k}$-lines of allowed electronic states. For the ZGNRs, the energy gap is determined by the edge states, and can be slightly regulated by uniaxial strain via variation of the population of edge states, but is robust under shear strain.

\section{Acknowledgements}

Y. L. thanks Bing Huang and Peng Wang for valuable discussions. This work was supported by the National Natural Science Foundation of China (Grants Nos. 50821061, 20973013), the Ministry of Science and Technology of China (Grants No. 2007CB936203), and 
the Fundamental Research Funds for the Central Universities.

Open Access: This article is distributed under the terms of the Creative Commons Attribution Noncommercial License which permits any noncommercial use, distribution, and reproduction in any medium, provided the original author(s) and source are credited.

\section{References}

[1] Geim, A. K.; Novoselov, K. S. The rise of graphene. Nat. Mater. 2007, 6, 183-191.

[2] Cresti, A.; Nemec, N.; Biel, B.; Niebler, G.; Triozon, F.; Cuniberti, G.; Roche, S. Charge transport in disordered graphene-based low dimensional materials. Nano Res. 2008, 1, 361-394.

[3] Yan, Q. M.; Huang, B.; Yu, J.; Zheng, F. W.; Zang, J.; Wu, J.; Gu, B. L.; Liu, F.; Duan, W. H. Intrinsic current-voltage characteristics of graphene nanoribbon transistors and effect of edge doping. Nano Lett. 2007, 7, 1469-1473.

[4] Novoselov, K. S.; Geim, A. K.; Morozov, S. V.; Jiang, D.; Zhang, Y.; Dubonos, S. V.; Grigorieva, I. V.; Firsov, A. A. Electric field effect in atomically thin carbon films. Science 2004, 306, 666-669.

[5] Biel, B.; Blase, X.; Triozon, F.; Roche, S. Anomalous doping effects on charge transport in graphene nanoribbons. Phys. Rev. Lett. 2009, 102, 096803.

[6] Pereira, V. M.; Neto, A. H. C. Strain engineering of graphene's electronic structure. Phys. Rev. Lett. 2009, 103, 046801.

[7] Ferralis, N.; Maboudian, R.; Carraro, C. Evidence of structural strain in epitaxial graphene layers on $6 \mathrm{H}-\mathrm{SiC}(0001)$. Phys. Rev. Lett. 2008, 101, 156801.

[8] Borysiuk, J.; Bozek, R.; Strupinski, W.; Wysmolek, A.; Grodecki, K.; Steapniewski, R.; Baranowski, J. M. Transmission electron microscopy and scanning tunneling microscopy investigations of graphene on $4 \mathrm{H}-\mathrm{SiC}(0001) . J$. Appl. Phys. 2009, 105, 023503.

[9] Sun, G. F.; Jia, J. F.; Xue, Q. K.; Li, L. Atomic-scale imaging and manipulation of ridges on epitaxial graphene on 6H-SiC(0001). Nanotechnology 2009, 20, 355701.

[10] Jun, S. Density-functional study of edge stress in graphene. Phys. Rev. B 2008, 78, 073405.

[11] Huang, B.; Liu, M.; Su, N. H.; Wu, J.; Duan, W. H.; Gu, B. L.; Liu, F. Quantum manifestations of graphene edge stress and edge instability: A first-principles study. Phys. Rev. Lett. 2009, 102, 166404.

[12] Lee, C.; Wei, X. D.; Kysar, J. W.; Hone, J. Measurement of the elastic properties and intrinsic strength of monolayer graphene. Science 2008, 321, 385-388.

[13] Mohiuddin, T. M. G.; Lombardo, A.; Nair, R. R.; Bonetti, A.; Savini, G.; Jalil, R.; Bonini, N.; Basko, D. M.; Galiotis, C.; Marzari, N.; Novoselov, K. S.; Geim, A. K.; Ferrai, A. C. Uniaxial strain in graphene by Raman spectroscopy: G peak splitting, Grüneisen parameters, and sample orientation. Phys. Rev. B 2009, 79, 205433.

[14] Ni, Z. H.; Yu, T.; Lu, Y. H.; Wang, Y. Y.; Feng, Y. P.; Shen, Z. X. Uniaxial strain on graphene: Raman spectroscopy study and band-gap opening. ACS Nano 2008, 2, 2301-2305.

[15] Kim, K. S.; Zhao, Y.; Jang, H.; Lee, S. Y.; Kim, J. M.; Kim, K. S.; Ahn, J. -H.; Kim, P.; Choi, J. -Y.; Hong, B. H. Large-scale pattern growth of graphene films for stretchable transparent electrodes. Nature 2009, 457, 706-710.

[16] Bao, W. Z.; Miao, F.; Chen, Z.; Zhang, H.; Jang, W. Y.; Dames, C.; Lau, C. N. Controlled ripple texturing of suspended graphene and ultrathin graphite membranes. Nat. Nanotechnol. 2009, 4, 562-566.

[17] Lee, M. L.; Fitzgerald, E. A.; Bulsara, M. T.; Currie, M. T.; Lochtefeld, A. Strained Si, SiGe, and Ge channels for highmobility metal-oxide-semiconductor field-effect transistors. J. Appl. Phys. 2005, 97, 011101.

[18] Yang, L.; Han, J. Electronic structure of deformed carbon nanotubes. Phys. Rev. Lett. 2000, 85, 154-157.

[19] Minot, E. D.; Yaish, Y.; Sazonova, V.; Park, J. Y.; Brink, M.; McEuen, P. L. Tuning carbon nanotube band gaps with strain. Phys. Rev. Lett. 2003, 90, 156401.

[20] Teague, M. L.; Lai, A. P.; Velasco, J.; Hughes, C. R.; Beyer, A. D.; Bockrath, M. W.; Lau, C. N.; Yeh, N. C. Evidence for strain-induced local conductance modulations in single-layer graphene on $\mathrm{SiO}_{2}$. Nano Lett. 2009, 9, 2542-2546.

[21] Chang, C. P.; Wu, B. R.; Chen, R. B.; Lin, M. F. Deformation effect on electronic and optical properties of nanographite ribbons. J. Appl. Phys. 2007, 101, 063506.

[22] Gui, G.; Li, J.; Zhong, J. X. Band structure engineering of graphene by strain: First-principles calculations. Phys. Rev. B 2008, 78, 075435.

[23] Farjam, M.; Rafii-Tabar, H. Comment on "Band structure engineering of graphene by strain: First-principles calculations". Phys. Rev. B 2009, 80, 167401.

[24] Gui, G.; Li, J.; Zhong, J. X. Reply to "Comment on 'Band structure engineering of graphene by strain: First-principles calculations.". Phys. Rev. B 2009, 80, 167402.

[25] Sun, L.; Li, Q. X.; Ren, H.; Su, H. B.; Shi, Q. W.; Yang, J. L. Strain effect on electronic structures of graphene nanoribbons: A first-principles study. J. Chem. Phys. 2008, 129, 074704.

[26] Pereira, V. M.; Neto, A. H. C.; Peres, N. M. R. Tight-binding approach to uniaxial strain in graphene. Phys. Rev. B 2009, 80,045401 . 
[27] Mohr, M.; Papagelis, K.; Maultzsch, J.; Thomsen, C. Twodimensional electronic and vibrational band structure of uniaxially strained graphene from $a b$ initio calculations. Phys. Rev. B 2009, 80, 205410.

[28] Hod, O.; Scuseria, G. E. Electromechanical properties of suspended graphene nanoribbons. Nano Lett. 2009, 9, 2619-2622.

[29] Alam, K. Uniaxial strain effects on the performance of a ballistic top gate graphene nanoribbon on insulator transistor. IEEE Trans. Nanotechnol. 2009, 8, 528-534.

[30] Pellegrino, F. M. D.; Angilella, G. G. N.; Pucci, R. Strain effect on the optical conductivity of graphene. Phys. Rev. B 2010, 81, 035411.

[31] Choi, S. M.; Jhi, S. H.; Son, Y. W. Effects of strain on electronic properties of graphene. Phys. Rev. B 2010, 81, 081407.

[32] Rasuli, R.; Rafii-Tabar, H.; Zad, A. I. Strain effect on quantum conductance of graphene nanoribbons from maximally localized Wannier functions. Phys. Rev. B 2010, 81, 125409.

[33] Poetschke, M.; Rocha, C. G.; Torres, L. E. F. F.; Roche, S.; Cuniberti, G. Modeling graphene-based nanoelectromechanical devices. Phys. Rev. B 2010, 81, 193404.

[34] Hossain, M. Z. Quantum conductance modulation in graphene by strain engineering. Appl. Phys. Lett. 2010, 96, 143118.

[35] Lu, Y.; Guo, J. Band gap of strained graphene nanoribbons. Nano Res. 2010, 3, 189-199.

[36] de Andres, P. L.; Verges, J. A. First-principles calculation of the effect of stress on the chemical activity of graphene. Appl. Phys. Lett. 2008, 93, 171915.

[37] Kresse, G.; Furthmüller, J. Efficiency of ab initio total energy calculations for metals and semiconductors using a plane-wave basis set. Comp. Mater. Sci. 1996, 6, 15-50.

[38] Kresse, G.; Joubert, D. From ultrasoft pseudopotentials to the projector augmented-wave method. Phys. Rev. B 1999, 59, 1758-1775.

[39] Perdew, J. P.; Wang, Y. Accurate and simple analytic representation of the electron-gas correlation-energy. Phys. Rev. B 1992, 45, 13244-13249.

[40] Harrison, W. A. Electronic structure and the properties of solids: The physics of the chemical bond; Dover Publications: New York, 1989.

[41] Ribeiro, R. M.; Pereira, V. M.; Peres, N. M. R.; Briddon, P. R.; Neto, A. H. C. Strained graphene: Tight-binding and density functional calculations. New J. Phys. 2009, 11, 115002.

[42] Castro Neto, A. H.; Guinea, F.; Peres, N. M. R.; Novoselov, K. S.; Geim, A. K. The electronic properties of graphene. Rev. Mod. Phys. 2009, 81, 109-162.

[43] Hasegawa, Y.; Konno, R.; Nakano, H.; Kohmoto, M. Zero modes of tight-binding electrons on the honeycomb lattice. Phys. Rev. B 2006, 74, 033413.

[44] Son, Y. W.; Cohen, M. L.; Louie, S. G. Energy gaps in graphene nanoribbons. Phys. Rev. Lett. 2006, 97, 216803.

[45] Saito, R.; Fujita, M.; Dresselhaus, G.; Dresselhaus, M. S. Electronic-structure of chiral graphene tubules. Appl. Phys. Lett. 1992, 60, 2204-2206.

[46] Mintmire, J. W.; Dunlap, B. I.; White, C. T. Are fullerene tubules metallic? Phys. Rev. Lett. 1992, 68, 631-634.

[47] Zheng, F. W.; Liu, Z. R.; Wu, J.; Duan, W. H.; Gu, B. L. Scaling law of the giant Stark effect in boron nitride nanoribbons and nanotubes. Phys. Rev. B 2008, 78, 085423.

[48] Wakabayashi, K.; Fujita, M.; Ajiki, H.; Sigrist, M. Electronic and magnetic properties of nanographite ribbons. Phys. Rev. B 1999, 59, 8271-8282.

[49] Son, Y. W.; Cohen, M. L.; Louie, S. G. Half-metallic graphene nanoribbons. Nature 2006, 444, 347-349.

[50] Zheng, F. W.; Zhou, G.; Liu, Z. R.; Wu, J.; Duan, W. H.; Gu, B. L.; Zhang, S. B. Half metallicity along the edge of zigzag boron nitride nanoribbons. Phys. Rev. B 2008, 78, 205415.

[51] Fujita, M.; Wakabayashi, K.; Nakada, K.; Kusakabe, K. Peculiar localized state at zigzag graphite edge. J. Phys. Soc. Jpn. 1996, 65, 1920-1923.

[52] Nakada, K.; Fujita, M.; Dresselhaus, G.; Dresselhaus, M. S. Edge state in graphene ribbons: Nanometer size effect and edge shape dependence. Phys. Rev. B 1996, 54, 17954-17961.

[53] Gunlycke, D.; Areshkin, D. A.; Li, J. W.; Mintmire, J. W.; White, C. T. Graphene nanostrip digital memory device. Nano Lett. 2007, 7, 3608-3611. 